\title{
TENDÊNCIAS DAS DESIGUALDADES DE ACESSO AO ENSINO SUPERIOR NO BRASIL: 1982-2010
}

\author{
ARnAldo LoPo Mont'Alvão NETO*
}

\begin{abstract}
RESUMO: Este trabalho analisa as tendências das desigualdades de acesso ao ensino superior no Brasil ao longo das últimas três décadas, período marcado por grande expansão do sistema de educação superior. São utilizadas informações da Pesquisa Nacional por Amostra de Domicílios (PNAD) e dos Censos Demográficos, a partir das quais modelos de regressão estimam as chances de estudantes que completaram o ensino médio chegarem ao ensino superior no país, em função das origens socioeconômicas, características sociodemográficas, estrutura familiar e diferenças regionais. Os principais resultados indicam queda das desigualdades de acesso, segundo nível educacional dos pais e diferenças raciais.
\end{abstract}

Palavras-chave: Ensino superior. Tendências. Desigualdades de acesso.

\section{INEQUALITIES IN ACCESS TO HIGHER EDUCATION IN BRAZIL: 1982-2010}

ABSTRACT: The paper aims to analyze recent trends regarding inequalities in the access to higher education in Brazil between 1982 and 2010, a period during which the educational system underwent massive expansion. It draws on data from the National Household Sample Survey (PNAD) and Demographic Censuses, based on which regression models estimate the chances of high school graduates achieving higher education as a function of their family background and structure, sociodemographic characteristics and geographical location. The main results indicate a decline in access inequalities related to racial differences and parents' education.

Key words: Higher education. Trends. Access inequalities.

Departamento de Sociologia da Universidade de Minnesota. Minneapolis (MN) - EUA. Contato com o autor: <almontalvao@gmail.com> 


\title{
INÉGALITÉ D'ACCÈS À L'ENSEIGNEMENT SUPÉRIEUR
}

\author{
AU BRÉSIL: 1982-2010
}

\begin{abstract}
RÉSUMÉ: Ce document examine les tendances d'inégalité d'accès à l'enseignement supérieur au Brésil au cours des trois dernières décennies, une période marquée par une grande expansion du système d'enseignement supérieur. Nous utilisons les informations de l'Enquête Nationale par échantillonnage Auprès des Ménages (PNAD) et recensements, dont les modèles de régression estiment les chances des étudiants qui ont conclu l'enseignement secondaire parviennent à l'enseignement supérieur en fonction de son origine socio-économique, socio-démographique, la structure familiale et les différences régionales. Les principaux résultats indiquent la baisse d'inégalité dans l'accès selon le niveau d'éducation des parents et des différences raciales.
\end{abstract}

Mots-clés: Enseignement supérieur. Tendances. Inégalités d'accès.

\section{Introdução}

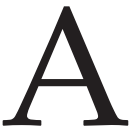

o longo das últimas décadas, sistemas educacionais têm se ampliado e um número cada vez maior de estudantes tem conseguido avançar aos níveis mais altos desses sistemas. Estimativas indicam que, enquanto em 1900 apenas $1 \%$ dos estudantes em idade apropriada estava matriculado em cursos superiores ao redor do mundo, atualmente este percentual é de $20 \%$, sendo que em alguns países - Japão e EUA, por exemplo - este percentual é igual ou maior que $60 \%$ (SCHOFER; MEYER, 2005; FRANK; MEYER, 2006). Uma série de fatores tem contribuído para isso. A melhoria geral das condições de vida das famílias (ERIKSON; JONSSON, 1996), fatores sociodemográficos como a extensão do período da juventude e adiamento do ingresso no mercado de trabalho (SCHWARTZMAN, 2005), assim como fatores estruturais como diferenciação institucional e aumento da participação do setor privado (PRATES, 2005), foram e têm sido determinantes para a expansão do acesso a este nível no Brasil e ao redor do mundo.

No entanto, apesar do aumento nas taxas de transição ao ensino superior, vários estudos apontaram que não houve grandes mudanças no que diz respeito às proporções de estudantes das diferentes origens sociais que conseguiram realizar esta transição, mantendo estáveis as desigualdades de acesso. A manutenção dessas diferenças dever-se-ia tanto a fatores no nível individual e familiar - recursos econômicos, culturais e sociais que o grupo familiar disponibiliza para as crianças e estas convertem em instrumentos de realização escolar (BOURDIEU, 1986) - quanto no nível estrutural - organização do sistema educacional e diferenças regionais, por exemplo -, fazendo com que grupos sociais tenham acessos diferenciados às oportunidades escolares. O volume Persistent inequality (SHAVIT; BLOSSFELD, 1993), por exemplo, analisou o caso de 13 países durante a década de 1980 e apontou que o 
processo de expansão educacional não resultou em diminuição das desigualdades na imensa maioria deles. Assim, ao contrário do que propuseram os teóricos da modernização (PARSONS, 1970; TREIMAN, 1970), expansão não levaria necessariamente ao arrefecimento imediato das desigualdades escolares.

Já a coletânea Stratification in higher education (SHAVIT; ARUM; GAMORAN, 2007) analisa o acesso à educação superior em 15 países e defende que o processo de expansão educacional, mesmo que não tenha reduzido desigualdades, é sim um processo inclusivo porque possibilitou que contingentes de jovens de famílias em desvantagem socioeconômica pudessem chegar a este nível de ensino. Breen e associados (2009), por sua vez, afirmam que tem havido sim queda na relação entre origens sociais e alcance educacional, principalmente na transição para a educação secundária, nível que intermedia a entrada para a educação superior, questionando a tese da desigualdade persistente.

A questão que motiva este estudo é entender as tendências de acesso ao ensino superior no Brasil ao longo das últimas três décadas, período marcado por grande expansão do sistema de ensino superior, por mudanças demográficas na população, além de políticas públicas implementadas com o intuito de diminuir as desigualdades de acesso. A seção a seguir discute as condições para redução destas desigualdades e, em seguida, são apresentados indicadores sobre a expansão do ensino superior no país. Posteriormente, são apresentadas as fontes de dados e a metodologia de análise, cujos resultados são discutidos em seguida. A seção final faz um apanhado destes resultados.

\section{Existem razões para acreditar num possível declínio do efeito das origens sociais sobre o acesso ao ensino superior no Brasil?}

As tendências apontadas pelos estudos mais recentes convergem com a tese da desigualdade persistente no Brasil, no que toca ao efeito das origens socioeconômicas (como classe social, renda e educação dos pais) - ver, por exemplo, Torche (2005), Collares (2010), Ribeiro (2011) e Mont'Alvão (2011). Por outro lado, desigualdades raciais, que eram estáveis até o início dos anos de 1990 (FERNANDES, 1999), começaram a declinar nos últimos anos (SCHWARTZMAN, 2004; COLLARES, 2010).

Parte da literatura internacional argumenta que a melhoria das condições de vida e mudanças demográficas nas últimas décadas tendem a provocar diminuição nas desigualdades educacionais (ERIKSON; JONSSON, 1996; BREEN et al., 2009). Fatores como a diminuição do tamanho das famílias, melhoria dos padrões de saúde e nutrição dos estudantes, expansão e melhoria dos sistemas de 
educação básica e de nível médio - principalmente no setor público, que tem facilitado o acesso de classes mais baixas -, ampliação da educação obrigatória, além da diminuição dos custos escolares e a diminuição da necessidade de estudantes ingressarem no mercado de trabalho para ajudar suas famílias, podem ter ajudado, em conjunto, a melhorar o rendimento de estudantes de classes trabalhadoras no sistema escolar. Outro fator importante é a mudança da própria composição das classes sociais, com diminuição das classes rurais ao longo do último século. Por fim, os processos de expansão e diversificação do próprio sistema de ensino superior teriam contribuído para atender um número maior de estudantes de diferentes origens sociais.

O Brasil não passou alheio a estes processos. Apesar da relativa estabilidade da estrutura de classes sociais no país entre os anos de 1970 e os 1990 (PASTORE; SILVA, 2000; RIBEIRO, 2003), a partir dos anos de 1970 aprofundaram-se as mudanças sociais, políticas e demográficas que criaram condições sociais mais favoráveis para a população em idade escolar básica. A diminuição do número de filhos por mulher, o adiamento do ingresso no mercado de trabalho, o aumento da densidade populacional nas cidades e maior cobertura do sistema escolar permitiram que as coortes mais jovens desfrutassem de um ambiente mais propício para a melhoria da realização escolar (SILVA; HASENBALG, 2000; SCHWARTZMAN, 2005). As melhorias na cobertura do sistema escolar fundamental e médio implicariam aumento da população jovem com possibilidades de entrar no ensino superior. Soma-se a isto a expansão do ensino superior e um processo incipiente, mas progressivo, de diversificação institucional, principalmente no setor privado, que tem levado ao aumento da proporção de matrículas de jovens entre 18 e 24 anos que ingressam no superior (PRATES, 2005; MONT'ALVÃO, 2013). ${ }^{1}$ A implementação de programas como Reestruturação e Expansão das Universidades Federais (Reuni), o Universidade para Todos (Prouni) e os programas de ação afirmativa ao longo da década oferecem um suporte adicional. ${ }^{2}$

Na medida em que estes processos tenham permitido que proporções cada vez maiores de jovens oriundos de estratos socioeconômicos mais baixos, assim como de grupos de cor (pretos e pardos), tenham acesso à educação superior, poderia esperar-se queda nas desigualdades.

\section{Indicadores da expansão da educação superior no Brasil}

O sistema educacional brasileiro tem apresentado melhoria importante de indicadores, com a educação básica atingindo uma cobertura superior a 96\% das crianças entre 7 e 14 anos e o ensino médio expandindo-se progressivamente (SILVA, 2003; SOARES; ALVES, 2003). O nível superior também se expandiu 
significativamente, principalmente na primeira década do século XXI, conforme ilustra a Figura 1. Entre 2001 e 2010, o número de matrículas mais do que dobrou, com um aumento de $110 \%$, atingindo 6,4 milhões de estudantes. A rede privada aumentou de 2,1 milhões para 4,7 milhões de matrículas (+126\%), elevando sua participação de $69 \%$ para $74 \%$ do total de matrículas, enquanto a rede pública, embora tenha aumentado de 944 mil para 1,6 milhões de estudantes (+74\%), teve uma diminuição na sua participação relativa de $31 \%$ para $26 \%$ das matrículas totais no período. ${ }^{3}$

\section{Figura 1}

Evolução das matrículas do ensino superior no Brasil entre 2001 e 2010



Fonte: Elaboração própria a partir de dados do Censo do Ensino Superior 2010 (INEP, 2011).

Em geral, o percentual da população em idade escolar adequada - 18 a 24 anos de idade - que tem acesso ao ensino superior também tem apresentado elevação nas últimas décadas, mas com maior intensidade na última, conforme mostra a figura 2. Entre a década de 1980 e o início da década de 1990 não houve aumento deste percentual, permanecendo próximo de 4,7\%. A partir do início dos anos 2000, esse percentual sobe para mais de $8 \%$ e atinge $14,4 \%$ em 2009 .

O aumento destes percentuais, é claro, só foi possível porque aumentou o número de estudantes elegíveis para ingresso no nível superior, que são aqueles que concluem o ensino médio. O número de matrículas no ensino médio aumentou progressivamente até o ano de 2005, declinando a partir daí, o que indica a diminuição das coortes de estudantes que concluem o ensino fundamental e redução da distorção entre idade é série no ensino médio (CORBUCCI, 2009). 
É importante considerar diferenças regionais. A tabela 1 fornece informações sobre a proporção de indivíduos matriculados em cada grande região brasileira, no início dos anos de 1980 e no final da última década. Ela indica que aumentou consideravelmente neste período a proporção de jovens que consegue acesso à educação superior em todas as regiões. Em média, o acesso foi triplicado, passando de $4,9 \%$ para $14,4 \%$ desta coorte, mas nas regiões Nordeste, Centro-Oeste e Sul o aumento foi de quatro vezes. Em seguida, mostra que a região Nordeste é aquela com menor taxa de acesso nos dois períodos, mas seguindo de perto a região Norte.

\section{Figura 2}

Evolução do percentual de estudantes entre 18 e 24 anos de idade com acesso ao ensino superior no Brasil entre 1982 e 2009

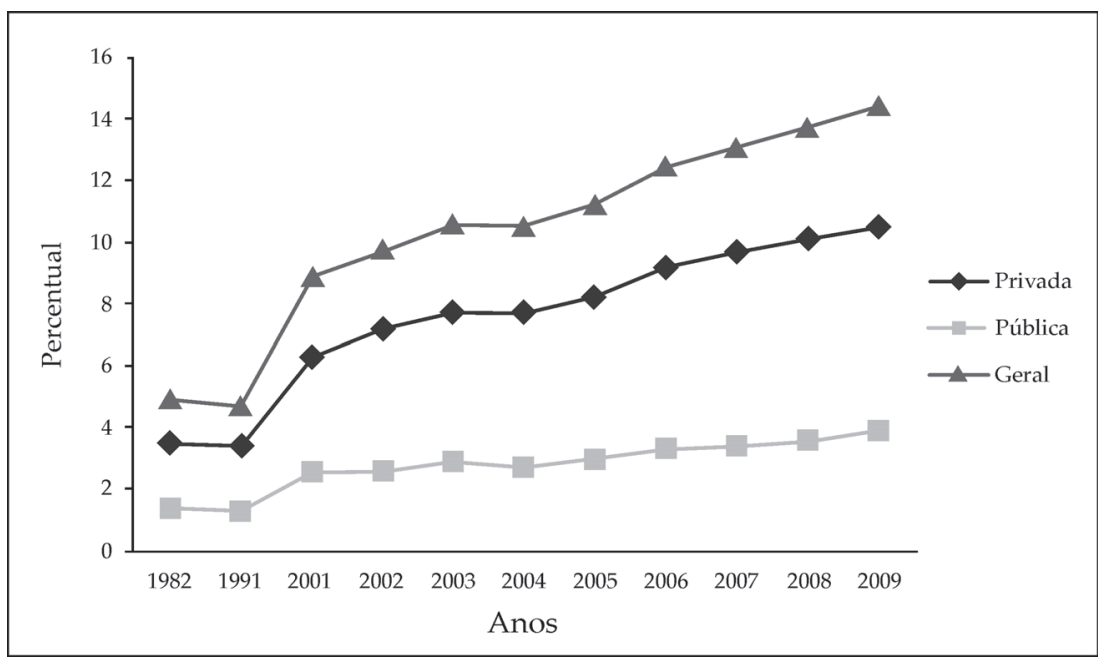

Fonte: PNAD (1982, 2001-2009) e Censo (1991).

Todavia, um dado importante para análise do acesso ao ensino superior, e que geralmente está ausente das análises, é que, apesar do aumento do número bruto de estudantes elegíveis, a proporção daqueles que realizam efetivamente a transição permanece praticamente inalterada, em torno de 35\%, entre o início dos anos de 1990 e o fim da última década, conforme indica a figura $3 .{ }^{4}$ Ao longo dos últimos 30 anos, uma proporção constante de praticamente dois terços dos estudantes entre 18 e 24 anos de idade que concluem o ensino médio não faz a transição para o ensino superior no Brasil. 


\section{Tabela 1}

Porcentagem de indivíduos entre 18 e 24 anos matriculados em instituições de ensino superior por região brasileira: 1982 e 2009

\begin{tabular}{|cc|c|}
\hline Regiões & Ano & Fizeram a transição \\
\hline \multirow{2}{*}{ NE } & 1982 & 2.4 \\
& 2009 & 9.2 \\
\hline \multirow{2}{*}{ N } & 1982 & 3.5 \\
& 2009 & 11 \\
\hline \multirow{2}{*}{ CO } & 1982 & 4 \\
& 2009 & 17.9 \\
\hline \multirow{2}{*}{ SE } & 1982 & 6.5 \\
& 2009 & 16.8 \\
\hline \multirow{2}{*}{ S } & 1982 & 5 \\
& 2009 & 19.2 \\
\hline \multirow{2}{*}{ Geral } & 1982 & 4.9 \\
& 2009 & 14.4 \\
\hline
\end{tabular}

Fonte: Elaboração própria a partir de dados da PNAD 1982 e 2009.

\section{Figura 3}

Percentual da população elegível que fez a transição para o ensino superior - Brasil: 1982 a 2010

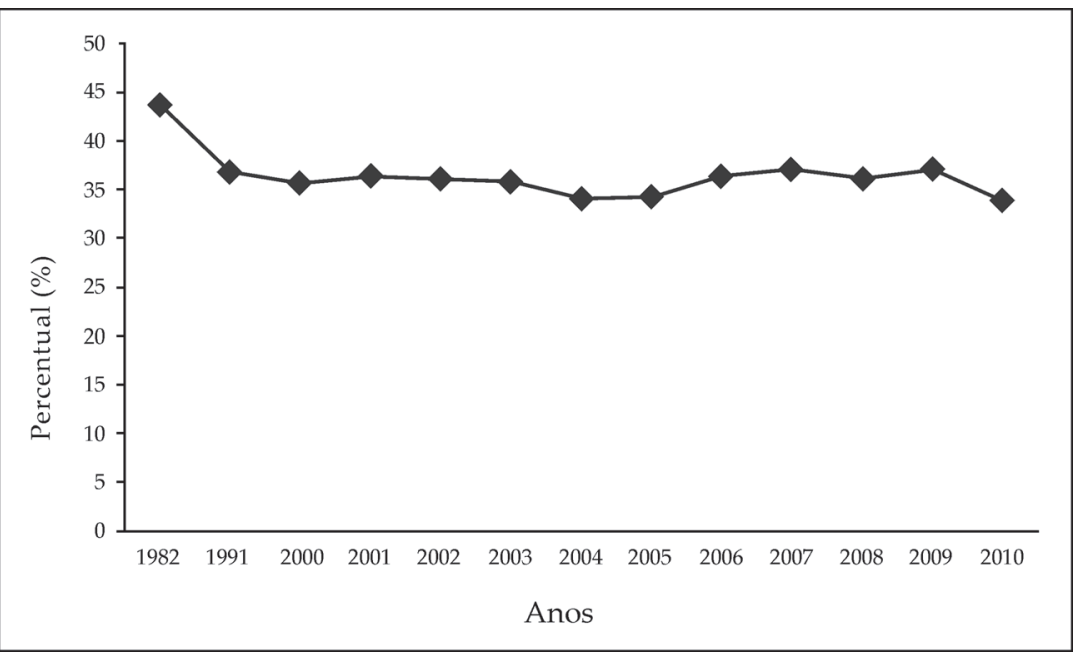

Fonte: Elaboração própria a partir de dados da PNAD (1982, 2001-2009) e dos Censos (1991, 2000, 2010). 
De acordo com Schwartzman (2004), três fatores de ordem institucional são determinantes para que a cobertura do sistema de ensino superior brasileiro seja tão limitada, mesmo comparando-se com outros países da América Latina. ${ }^{5} \mathrm{O}$ primeiro é o filtro imposto pelo ensino médio, que apesar de apresentar grande expansão, ainda não está próximo do processo de universalização. Em 2002, somente $40 \%$ dos estudantes entre 15 e 17 anos estavam matriculados neste nível, e em 2009, segundo dados da PNAD, subiu para $51 \%$. O segundo fator seria o caráter seletivo do sistema superior. Entre aqueles que conseguem passar pelo filtro do ensino médio, a competição através dos vestibulares e outras formas de ingresso elimina grande parte dos candidatos. O terceiro diz respeito ao alto custo do sistema público, o qual limita suas possibilidades de expansão. Um quarto fator implícito é a rigidez organizacional do sistema, com ainda pouca abertura a outros formatos institucionais e pedagógicos, como aqueles mais voltados para o mercado de trabalho, principalmente para uma formação técnica com menor duração.

\section{Análise da evolução das desigualdades de acesso ao ensino superior entre 1982 e 2010}

\section{Fontes de dados}

O foco principal desta análise é o período que corresponde à primeira década deste século e, para tal, são utilizadas informações da Pesquisa Nacional por Amostra de Domicílios (PNAD) de 2001 a 2009. Para estender esse período e incluir os anos 2000 e 2010 são utilizados dados dos Censos Demográficos destes anos. E para proporcionar referências de contraste com as décadas anteriores foram incluídas informações da PNAD de 1982 e do Censo Demográfico de 1991. Assim, as análises cobrem um período de aproximadamente três décadas, entre 1982 e 2010.

Estas duas fontes trazem informações similares sobre as características familiares, demográficas e socioeconômicas dos indivíduos. Todavia, para respeitar as propriedades amostrais de cada uma, os modelos são construídos separadamente. São analisados apenas indivíduos entre 18 e 24 anos de idade - cuja condição na família é filho(a), a fim de se poder estimar o efeito da origem social (variáveis dos pais) sobre o alcance educacional - e que tenham completado o ensino médio.

\section{Subamostras}

A tabela 2 traz o tamanho das amostras analisadas em cada ano, incluindo-se as proporções dos que conseguiram fazer a transição. 
Tabela 2

Subamostras das PNAD e Censos Demográficos de 1982 a 2010.

\begin{tabular}{|cccc|}
\hline Ano & $\begin{array}{c}\text { Tamanho da } \\
\text { Subamostra }\end{array}$ & $\begin{array}{c}\text { Deixaram o sistema } \\
\text { escolar }(\%)\end{array}$ & $\begin{array}{c}\text { Fizeram a } \\
\text { transição (\%) }\end{array}$ \\
\hline 1982 & 6730 & 56.3 & 43.7 \\
1991 & 200485 & 63.1 & 36.9 \\
2000 & 436042 & 64.3 & 35.7 \\
2001 & 9825 & 63.6 & 36.4 \\
2002 & 11260 & 63.9 & 36.1 \\
2003 & 11876 & 64.2 & 35.8 \\
2004 & 12653 & 66.0 & 34 \\
2005 & 13459 & 65.8 & 34.2 \\
2006 & 14204 & 63.6 & 36.4 \\
2007 & 13902 & 62.9 & 37.1 \\
2008 & 14125 & 63.9 & 36.1 \\
2009 & 14206 & 62.9 & 37.1 \\
2010 & 686684 & 66.1 & 33.9 \\
\hline & Média & 63.6 & 36.4 \\
\hline
\end{tabular}

\section{Métodos de análise}

Serão utilizados modelos de regressão logística binária, os quais analisam as chances de indivíduos com determinadas características fazerem a transição para o ensino superior. Para controle de possível viés de seletividade - em cada transição são analisados aqueles que foram selecionados na transição anterior e, então, quanto mais alta a transição, maior o viés - e da ausência de informações importantes - como o nível de desempenho acadêmico e de habilidades cognitivas, que geralmente não estão disponíveis em pesquisas domiciliares, e cuja ausência tende a provocar viés nos coeficientes relacionados a origens sociais (CAMERON; HECKMAN, 1998) -, são empregadas classes latentes, estratégia utilizada por alguns trabalhos (CAMERON; HECKMAN, 1998; TAM, 2011) para lidar com estas limitações. Com esta abordagem são identificadas características latentes (não observadas) a partir das variáveis incluídas nos modelos, e que possibilitam agrupar estes indivíduos em classes (clusters) com propensões a fazerem as mesmas escolhas educacionais. Os modelos aqui estimados procuram identificar duas grandes classes latentes a partir das variáveis observadas: uma para indivíduos com maior 
propensão de chegar ao ensino superior, e outra para indivíduos com características que levem a uma menor propensão de fazer a transição. Os modelos finais levam em conta estas diferenças na propensão de cada classe latente para realização da transição.

A equação geral para modelos deste tipo é:

$$
\log \left(\frac{P_{k}}{1-P_{i k}}\right)=\beta_{0 k}+\beta_{1 k} x_{i l}+\cdots+\beta_{j k} x_{j}+\beta_{u k} U
$$

Onde a probabilidade $\left(\frac{P_{k}}{1-P_{i k}}\right)\left(\frac{P_{k}}{1-P_{i k}}\right)$ de um indivíduo $i$ completar a transição $k$ depende do efeito das origens socioeconômicas $\left.\left(x_{i l}\right) x_{i l}\right)$, variáveis de controle $\left(x_{j}\right)$ $x_{j}$ ), e heterogeneidade não observada $\left.(U) \cdot U\right)$.

Resultados obtidos com estes modelos serão comparados com resultados de modelos que não fazem este tipo de controle, com o intuito de mostrar as possíveis limitações destes últimos, as quais podem ter consequências para a interpretação de resultados de estudos anteriores neste campo.

\section{Variáveis}

As variáveis utilizadas nos modelos indicam:

- Características sociodemográficas: raça (variáveis indicadoras para amarelos, brancos, pardos e pretos) e gênero (variável binária);

- Origens socioeconômicas: nível educacional da mãe ou responsável (quatro variáveis indicadoras para $4^{\mathrm{a}}$ série, $8^{\mathrm{a}}$ série, ensino médio e ensino superior) e classe social do pai ou responsável - esquema EGP (ERIKSON; GOLDTHORPE, 1992) - com oito classes: profissionais de alto nível (I), profissionais de baixo nível (II), trabalhadores não manuais de rotina (IIIa), pequenos proprietários com e sem empregados (IVab), fazendeiros e trabalhadores rurais autoempregados (IVc), trabalhadores manuais qualificados $(\mathrm{V}+\mathrm{VI})$, trabalhadores manuais não qualificados urbanos (VIIa) e trabalhadores manuais não qualificados rurais (VIIb)];

- Estrutura familiar: uma variável binária para família monoparental e uma variável para o número de irmãos do estudante que vivem no domicílio;

- Participação no mercado de trabalho: com os dados da PNAD estima-se o efeito da participação no mercado de trabalho durante a educação básica (variável binária), e com os dados dos Censos é estimado o efeito da participação atual no mercado de trabalho (variável binária); 
- Diferenças regionais: variáveis indicadoras para as cinco grandes regiões do país.

\section{Resultados}

A tabela 3 traz indicadores do ajuste dos modelos e mostra que o modelo de classes latentes (CL), tanto para os dados da PNAD quanto para os do Censo, ao apresentar $R^{2}$ um pouco maior e menor $\log$ likelihood, se ajusta melhor aos dados que aqueles dos modelos logísticos binários (MLB) que não fazem controle. $\mathrm{O} R^{2}$ indica que, para o período de 2001 a 2009, as variáveis incluídas explicam, em média, pouco mais de $22 \%$ da variação total do modelo, enquanto para os dados dos censos de 1991 a 2010 esse valor é pouco maior que 18\%.

\section{Tabela 3}

Indicadores de ajuste dos modelos de regressão

\begin{tabular}{|l|cc|cc|}
\hline \multirow{2}{*}{ Modelos } & \multicolumn{2}{|c|}{ PNAD 2001-2009 } & \multicolumn{2}{c|}{ Censo 1991-2010 } \\
& (-2) Log likelihood & Pseudo $R^{2}$ & (-2) Log likelihood & Pseudo $R^{2}$ \\
\hline MLB & 97266 & 0.221 & 117501 & 0.181 \\
CL & 97144 & 0.222 & 117342 & 0.184 \\
\hline
\end{tabular}

Fonte: Elaboração própria a partir de dados das PNAD (1982 e 2001-2009) e dos Censos (1991, 2000 e 2010).

A discussão dos coeficientes obtidos nos modelos de regressão é feita a partir de gráficos que ilustram as tendências das desigualdades entre 1982 e $2010 .{ }^{6}$ Primeiro serão mostrados indicadores relativos às classes latentes estimadas e, em seguida, os gráficos segundo cada dimensão analisada.?

\section{Classes latentes}

A tabela 4 traz os indicadores gerais relativos às duas classes latentes detectadas pelos modelos de regressão. A classe 1 abriga a menor parte dos estudantes: $15,6 \%$, enquanto a classe 2 , por sua vez, abriga a maioria: $84,4 \%$. Estudantes da classe 1 , todavia, têm chances muito maiores de fazer a transição para o ensino superior do que aqueles da classe $2: 64 \%$ contra $24 \%$. A interpretação é que a classe latente 2 seria aquela com menor nível de desempenho acadêmico e habilidades cognitivas, enquanto a classe 1 representaria indivíduos com melhor desempenho acadêmico, que obteriam maior sucesso nos exames vestibulares. 


\section{Tabela 4}

Indicadores de pertencimento às classes latentes: 1982 a 2010

\begin{tabular}{|l|c|c|}
\hline \multicolumn{1}{|c|}{ Indicadores de pertencimento às classes latentes } & Classe 1 & Classe 2 \\
\hline Probabilidade geral de pertencimento & 0.156 & 0.844 \\
Probabilidade de caminho de acordo com a classe latente & & \\
Sair do sistema educacional & 0.362 & 0.760 \\
Fazer a transição & 0.638 & 0.240 \\
\hline
\end{tabular}

Fonte: Elaboração própria a partir de dados da PNAD (1982 e 2001-2009) e dos Censos (1991, 2000 e 2010).

\section{Características sociodemográficas}

\section{Gênero}

A figura 4 mostra que mulheres apresentam probabilidade muito maior que homens de fazer a transição para a educação superior no Brasil. Mostra também que esta vantagem já existia nos anos de 1980 e vem aumentando progressivamente. Assim, o Brasil segue a tendência mundial de vantagem feminina de acesso a este nível de ensino. No caso brasileiro esta vantagem configurou-se antes da média mundial (RIBEIRO, 2009), já que na maioria dos países homens ainda tinham vantagem de acesso até o início dos anos de 1990 (SCHOFER; MEYER, 2005).

O modelo de classes latentes indica que o efeito é em geral maior que aquele geralmente estimado em modelos que não controlam pela heterogeneidade não observada.

\section{Raça}

Os coeficientes ilustrados na figura 5 indicam que pardos, mas, principalmente, brancos e amarelos, apresentam vantagens de acesso ao ensino superior no Brasil em relação a pretos, mesmo controlando-se pelas origens sociais e diferenças regionais. Os coeficientes dos modelos com classes latentes são também maiores para esta dimensão.

As tendências indicam que, apesar da estabilidade do efeito para pardos, tem havido diminuição do efeito para brancos e amarelos, ou seja, tem ocorrido redução das desigualdades para esta dimensão. 


\section{Figura 4}

Tendências do efeito do gênero sobre as chances de acesso ao ensino superior no Brasil, 1982 a 2010

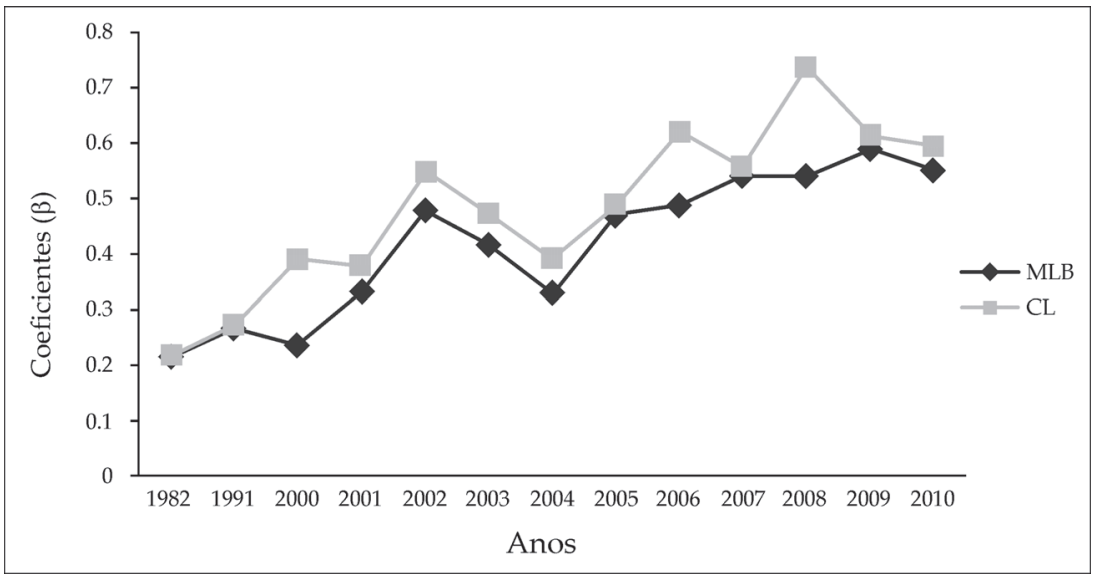

Fonte: Elaboração própria a partir de dados das PNAD (1982 e 2001-2009) e dos Censos (1991, 2000 e 2010).

\section{Figura 5}

Efeito das diferenças raciais na transição para a educação superior no Brasil: 1982 a 2010 (referência: pretos)

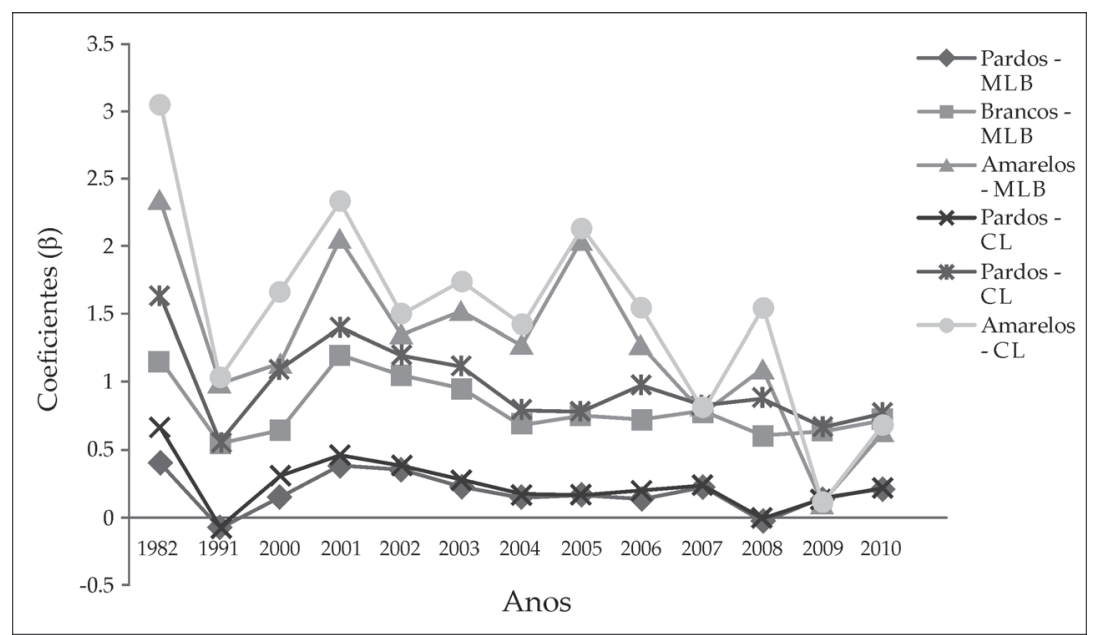

Fonte: Elaboração própria a partir de dados das PNAD (1982 e 2001-2009) e dos Censos (1991, 2000 e 2010). 


\section{Origens socioeconômicas}

As diferenças médias nas probabilidades de acesso por classe social e nível educacional dos pais estão ilustradas nas figuras 6 e 7, respectivamente. O modelo de classes latentes mais uma vez indica que os coeficientes são maiores quando se controla a heterogeneidade. As classes profissionais, I e II, são aquelas com maior probabilidade de acesso, com, respectivamente, 2,5 vezes e duas vezes mais chances de acesso que estudantes cujos pais situam-se na classe dos trabalhadores rurais não qualificados (VIIb). Em seguida, temos estudantes cujos pais são pequenos proprietários (IVab), com $80 \%$ a mais de chances, filhos de trabalhadores não manuais de rotina (IIIa), com $60 \%$ de vantagens, e filhos de pequenos proprietários rurais, com cerca de $30 \%$ de vantagens. Estudantes cujos pais são trabalhadores manuais urbanos qualificados (V+VI), ou não qualificados (VIIa), não apresentam vantagens estatisticamente significativas em relação a filhos de trabalhadores rurais. Interessante notar então que não existem diferenças entre filhos de trabalhadores manuais qualificados e não qualificados urbanos e filhos de trabalhadores não qualificados no meio rural.

\section{Figura 6}

Efeito médio da classe social dos pais na transição para a educação superior no Brasil: 2001 a 2009 (referência: trabalhadores rurais não qualificados - VIIb)

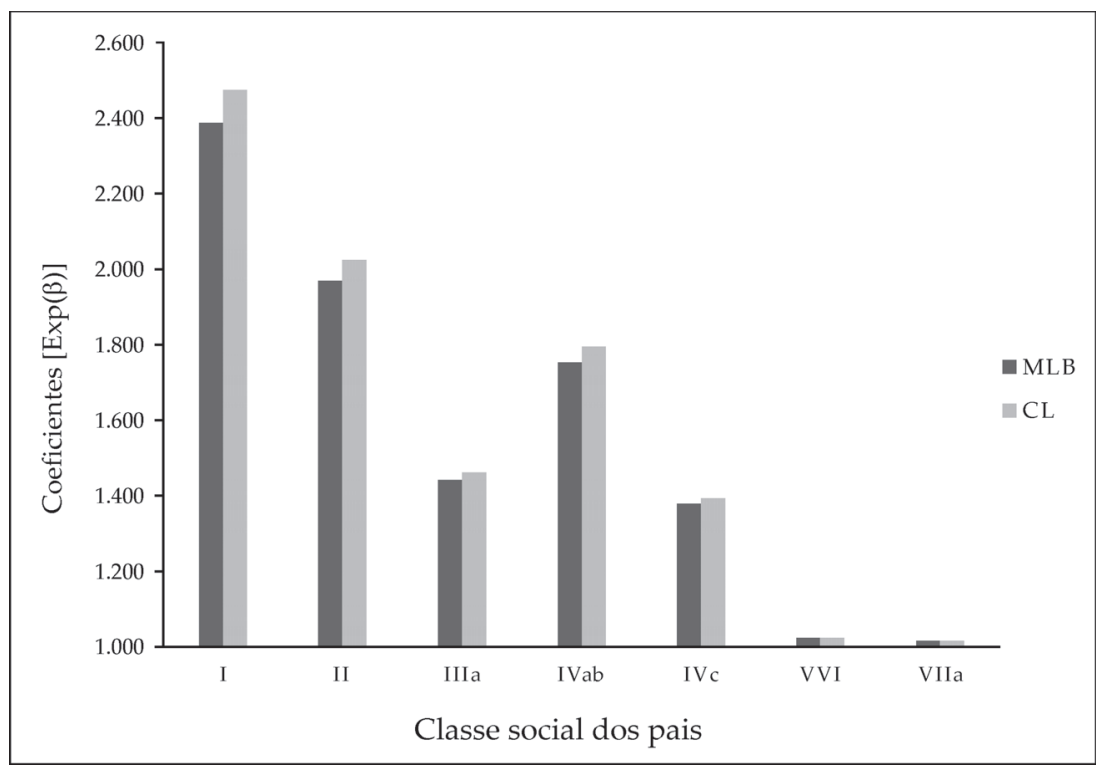

Fonte: Elaboração própria a partir de dados das PNAD (2001-2009).

No que concerne à educação dos pais, coeficientes indicam que estudantes cujos pais completaram apenas o ensino fundamental têm duas vezes mais chances 
de chegar ao ensino superior do que aqueles cujos pais não têm nenhum nível formal de escolaridade. Já aqueles cujos pais cursaram ensino médio têm quatro vezes mais chances, e aqueles cujos pais tiveram acesso ao ensino superior têm 16 vezes mais chances de chegar a esse nível de ensino. É possível interpretar esta enorme diferença a partir da ideia de que o nível educacional dos pais funciona como um piso mínimo de realização educacional para os filhos (MARE; CHANG, 2006), fazendo com que aqueles cujos pais cursaram o ensino superior tenham chances muito superiores de chegar ao mesmo nível educacional.

\section{Figura 7}

Efeito médio do nível de escolaridade dos pais na transição para a educação superior no Brasil: 2001 a 2009 (referência: estudantes cujos pais não têm nenhuma escolaridade formal completa)

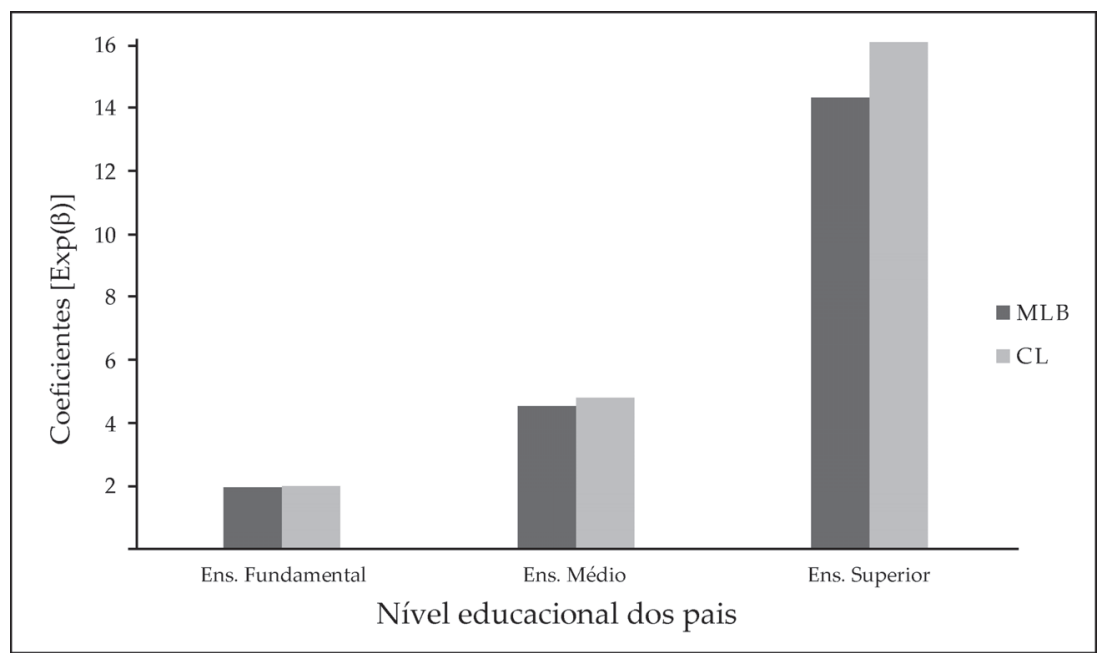

Fonte: Elaboração própria a partir de dados das PNAD (2001-2009).

A evolução destes efeitos ao longo do período estudado é indicada nas figuras 8 e 9. Para a classe social, apesar de ondulações pontuais, verifica-se, em geral, uma estabilidade entre 1982 e 2010, indicando que as desigualdades de classe social têm sido resistentes ao processo de expansão educacional e outras mudanças estruturais, como melhoria das condições de vida em geral.

Já a figura 9 indica que tem havido diminuição das desigualdades relacionadas ao nível escolar dos pais na última década, principalmente a partir de 2005. Assim, embora as desigualdades relacionadas à situação econômica da família e a capacidade efetiva das famílias de investir na educação dos filhos, indicadas pela classe social, sejam resistentes às transformações ocorridas nas últimas décadas, àquelas correspondentes aos recursos culturais e na medida em que eles valorizam 
e incentivam a progressão educacional dos filhos, indicadas pela educação dos pais, têm apresentado queda, proporcionando mais chances de acesso ao ensino superior a indivíduos cujos pais não tiveram oportunidades no passado. Em outras palavras, embora os pais com menor nível educacional tenham passado a incentivar mais os filhos a chegar ao nível superior, a estabilidade do efeito da classe indica a persistência das diferenças na capacidade das famílias bancarem na prática a educação dos filhos, independentemente do nível educacional dos pais.

\section{Figura 8}

Evolução do efeito da classe social dos pais na transição para a educação superior no Brasil: 1982 a 2010 (referência: trabalhadores rurais não qualificados - VIIb)

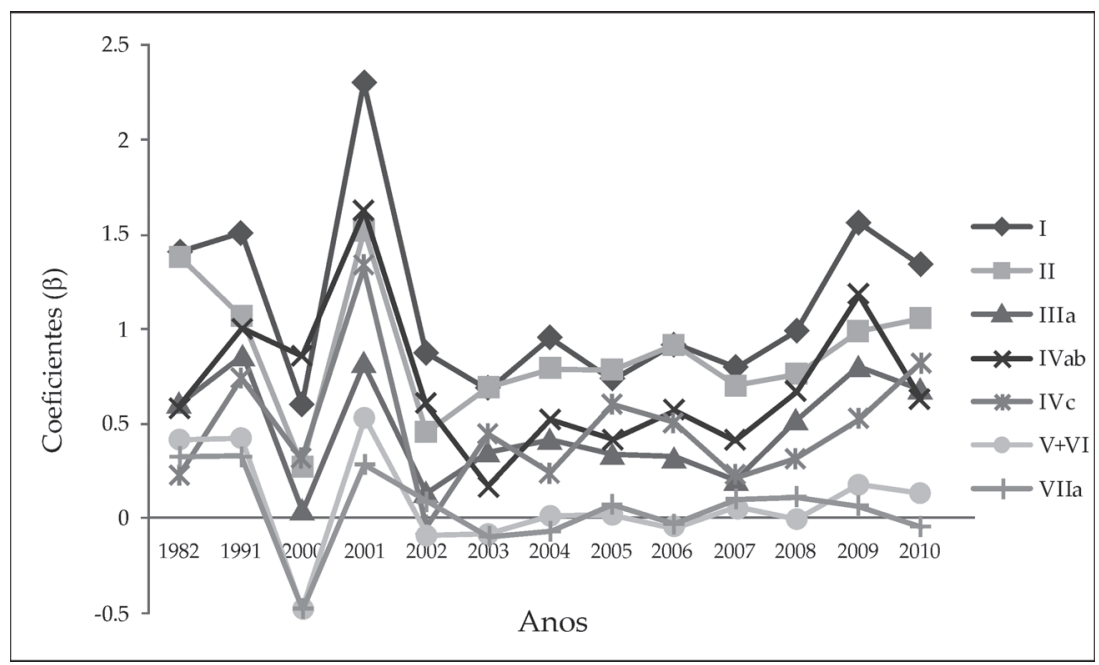

Fonte: Elaboração própria a partir de dados das PNAD (1982 e 2001-2009) e dos Censos (1991, 2000 e 2010).

\section{Estrutura familiar}

Esta dimensão indica a intensidade da interação entre estudantes e seus pais (DOWNEY, 1995). Estudantes que vivem com apenas um dos pais, e/ou em famílias com grande número de irmãos, têm desvantagens em suas chances de completar a transição. Ser criado com apenas um dos pais implica, em média, 35\% menos chances de chegar ao ensino superior em relação àqueles que viveram com os dois pais. Cada irmão a mais diminui em $15 \%$ estas chances.

As figuras 10 e 11 ilustram estes efeitos ao longo do período e apontam que não houve mudança significativa. 


\section{Figura 9}

Evolução do efeito do nível educacional dos pais na transição para a educação superior no Brasil, 1982 a 2010 (referência: estudantes cujos pais não têm escolaridade formal completa)

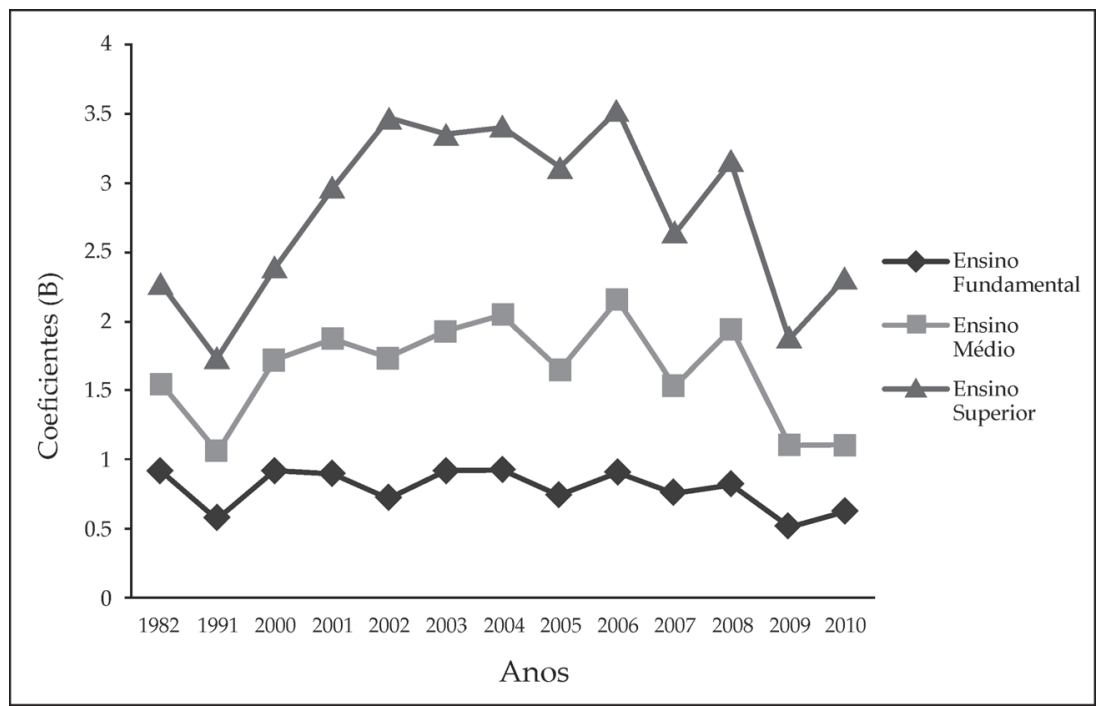

Fonte: Elaboração própria a partir de dados das PNAD (1982 e 2001-2009) e dos Censos (1991, 2000 e 2010).

Figura 10

Evolução do efeito da família monoparental nas chances de estudantes fazerem a transição para a educação superior no Brasil: 1982 a 2010

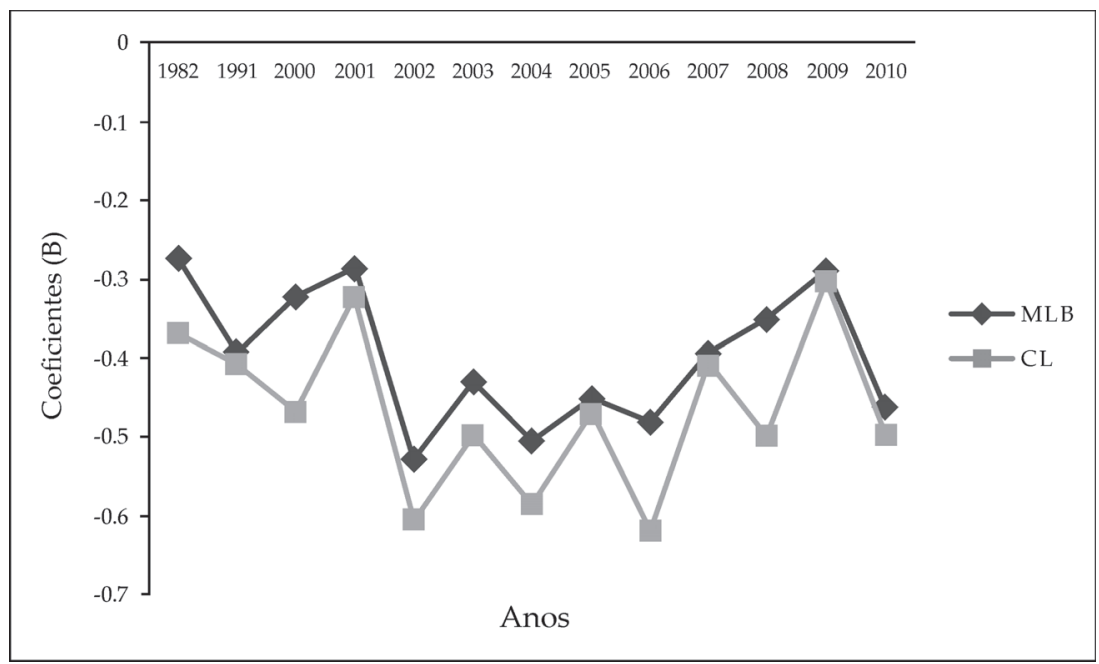

Fonte: Elaboração própria a partir de dados das PNAD (1982 e 2001-2009) e dos Censos (1991, 2000 e 2010). 


\section{Figura 11}

Evolução do efeito do número de irmãos nas chances de estudantes fazerem a transição para a educação superior no Brasil: 1982 a 2010

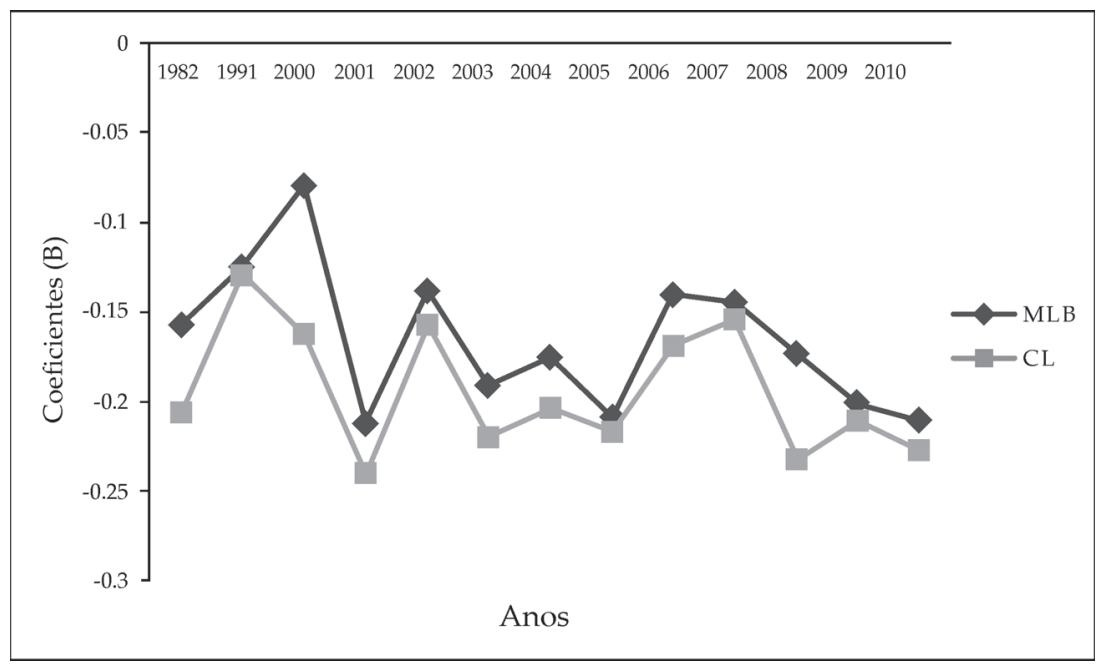

Fonte: Elaboração própria a partir de dados das PNAD (1982 e 2001-2009) e dos Censos (1991, 2000 e 2010).

\section{Inserção no mercado de trabalho}

\section{Trabalho durante a educação básica}

Indivíduos que começaram a trabalhar durante a educação básica têm menores chances de completar a transição para a educação superior, independentemente da origem socioeconômica, e este efeito diminui muito pouco ao longo do período, conforme ilustra a figura 12. Isto indica que estudantes que tiveram de dividir seus esforços entre trabalho e estudo durante a educação básica, muitos dos quais têm que frequentar cursos noturnos que contribuem pouco para sua formação (SCHWARTZMAN, 2005), ou não conseguem ser aprovados nos exames vestibulares ou decidem investir seus esforços no mercado de trabalho e não buscam a educação superior após completarem o ensino médio.

\section{Trabalho atual}

O efeito de estar trabalhando atualmente também é, em geral, negativo para as chances de acesso ao ensino superior. No entanto, conforme pode ser visto na figura 13, tem havido uma diminuição progressiva deste efeito. Esta relação indica 
que estudantes que trabalham têm procurado cada vez mais conciliar esta atividade com estudos superiores.

\section{Figura 12}

Evolução do efeito da participação prévia no mercado de trabalho sobre as chances de estudantes fazerem a transição para a educação superior no Brasil: 2001 a 2009

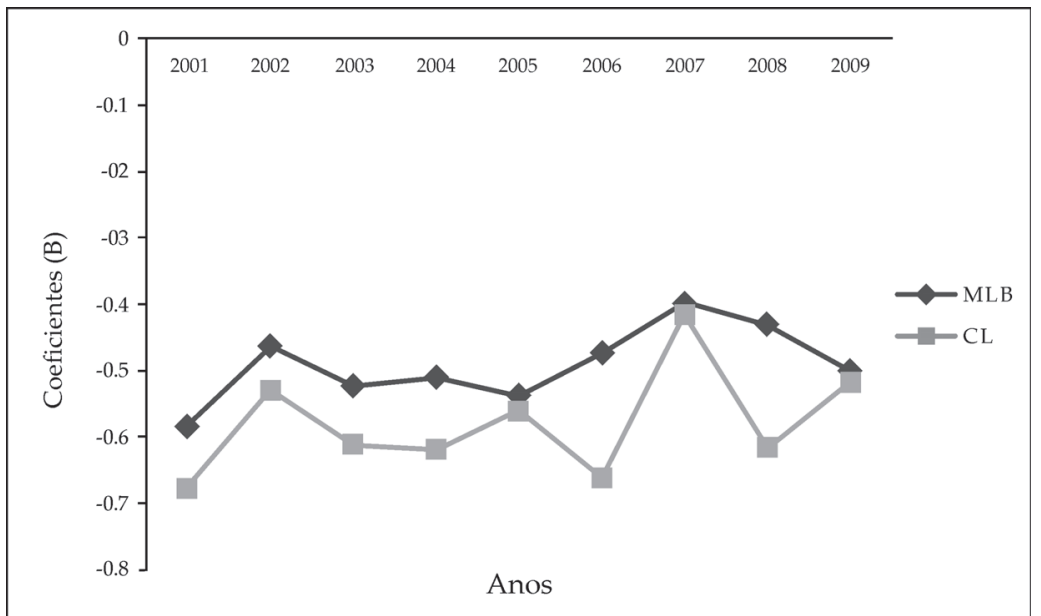

Fonte: Elaboração própria a partir de dados das PNAD (2001-2009).

\section{Figura 13}

Evolução do efeito da participação atual no mercado de trabalho sobre as chances de estudantes fazerem a transição para a educação superior no Brasil: 1982 a 2010



Fonte: Elaboração própria a partir de dados da PNAD (1982) e dos Censos (1991, 2000 e 2010). 


\section{Diferenças entre regiões}

Os coeficientes ilustrados na figura 14 indicam que estudantes das regiões Sul e Centro-Oeste são, em geral, aqueles com maiores probabilidades de acesso ao ensino superior no país, tendo como referência a região Nordeste. Em seguida vêm os da região Sudeste. Enquanto as vantagens de estudantes desta última região aumentaram a partir de 2004, entre as duas primeiras o efeito é estável ao longo da última década. Estudantes da região Norte passaram a apresentar vantagens a partir de 2009, mas sem uma tendência definida, de forma que em 2010 as diferenças eram próximas de zero.

\section{Figura 14}

Evolução das probabilidades de estudantes fazerem a transição para a educação superior por região do Brasil: 1982 a 2010

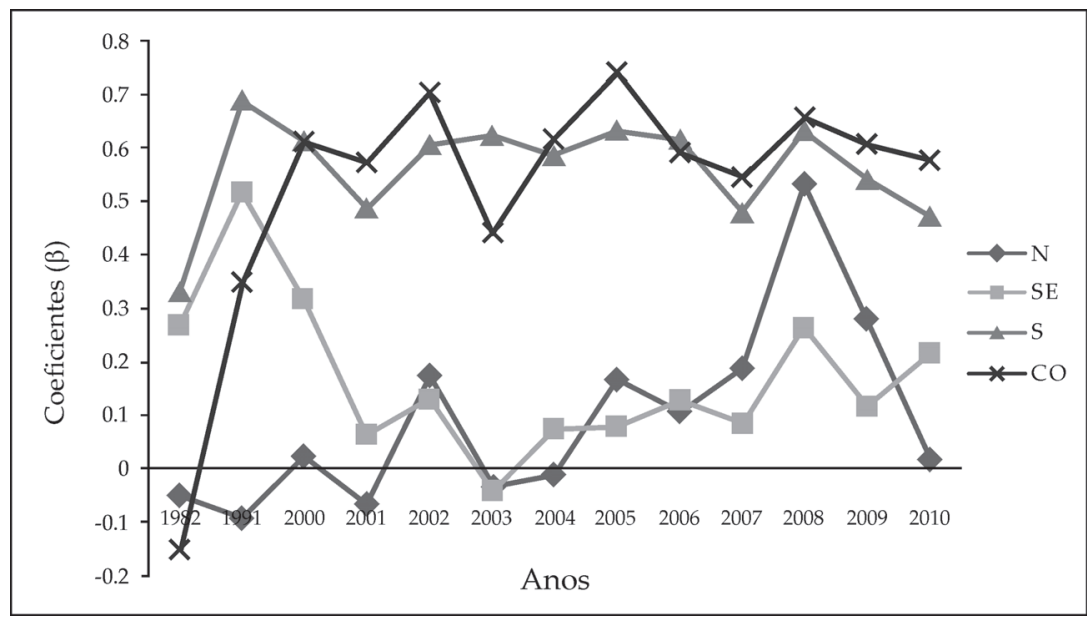

Fonte: Elaboração própria a partir de dados das PNAD (1982 e 2001-2009) e dos Censos (1991, 2000 e 2010).

\section{Discussão}

Este estudo analisou as tendências das desigualdades de acesso ao ensino superior no Brasil ao longo das últimas três décadas, utilizando dados da PNAD e dos Censos Demográficos, a partir dos quais foram estimados modelos de regressão logística com controle de heterogeneidade não observada - que possibilita minimizar o impacto do viés de seleção e da ausência de informações como níveis de desempenho acadêmico e habilidade cognitiva. Em geral, os resultados obtidos através destes modelos indicam que estudos que utilizam a PNAD como fonte de 
informação e não controlam pela heterogeneidade não observada tendem a subestimar as desigualdades de acesso ao ensino superior.

Os resultados indicam ainda que, mesmo minimizando estes problemas, as chances de estudantes chegarem ao ensino superior são influenciadas pelas origens socioeconômicas, pela estrutura familiar, pelo grupo racial, gênero, inserção no mercado de trabalho e localização regional. As tendências observadas apontam para a estabilidade, entre 1982 e 2010, do efeito da classe social e da estrutura familiar, e para aumento das vantagens das mulheres. Por outro lado, indicam a diminuição das desvantagens de estudantes que precisam ou escolhem trabalhar, assim como de grupos de cor e de estudantes cujos pais têm alcance educacional limitado, ou seja, é possível afirmar que tem havido redução das desigualdades nestas três dimensões nos últimos anos. A expansão do sistema e dos cursos noturnos, que permitem aos estudantes trabalhar e frequentar cursos superiores, a melhoria das condições para os grupos de cor, assim como o maior incentivo de pais com pouca escolaridade, têm feito diferença significativa no que concerne ao acesso a este nível.

Fica em aberto, para os próximos anos, uma análise do impacto geral da abertura do sistema público, determinada pelo Projeto de Lei n. 180, assim como da contínua disponibilização de bolsas de estudos na rede privada através do Prouni.

\section{Notas}

1. Dois argumentos importantes contrapõem-se no debate sobre as políticas que estruturam os rumos da educação superior no Brasil. O primeiro propõe que a expansão da rede pública é o caminho mais apropriado para se chegar à democratização do acesso a este nível (DURHAN, 2004; MARTINS, 2009), ao passo que o segundo sugere que se intensifique o processo de diferenciação institucional, investindo-se mais em educação vocacional e expandindo-se a política de crédito educativo (PRATES, 2005; SCHWARTZMAN, 2005). Para um aprofundamento desta discussão, consultar Mont'Alvão (2013).

2. Os programas de ação afirmativa tiveram início no Brasil no começo da década passada e vêm proporcionando vantagens para um número cada vez maior de estudantes de grupos em desvantagem socioeconômica, assim como grupos de cor terem acesso ao ensino superior. No entanto, apesar do apoio político, estes programas encontram certa resistência no meio intelectual, que defende o uso de critérios socioeconômicos para democratização do acesso, mas rechaça critérios raciais, pois estes tenderiam a intensificar divisões raciais e produzir discriminação às avessas - ver, por exemplo, Reis (2004) e Maggie e Fry (2004).

3. A forte expansão do setor privado no Brasil - que encontra processo similar apenas em alguns países da América Latina (Colômbia, Peru e México) e da Ásia (Taiwan, China, Vietnã, etc.) - e as dificuldades de regulação culminaram num setor gigantesco, voltado basicamente para atividades de ensino, e que atraem estudantes mais velhos que a média, os quais têm que conciliar estudos com o trabalho, o que ajuda a explicar a grande quantidade de cursos noturnos (CASTRO, 2004).

4. Este percentual é de $40 \%$ na Suíça, $65 \%$ nos EUA e Japão, 90\% no Reino Unido e na Itália.

5. Conforme indicado no estudo de Shavit et al. (2007), os países que apresentam as maiores taxas de acesso ao ensino superior são aqueles com sistemas mais diversificados, com maior abertura organizacional para acomodar os cursos técnicos e tecnológicos.

Educ. Soc., Campinas, v. 35, n. 127, p. 417-441, abr.-jun. 2014 
6. As tabelas com os coeficientes não foram inseridas neste trabalho, para evitar repetição de resultados.

7. Os gráficos trazem os coeficientes $(\beta)$ obtidos nos modelos de regressão, de forma que pontos acima do marco zero no eixo $y$ indicam efeito positivo, enquanto abaixo indicam efeito negativo, em relação à categoria de referência.

\section{Referências}

BOURDIEU, P. The forms of capital. In: RICHARDSON, J. (Ed.). Handbook of theory and research for the sociology of education. New York: Greenwood, 1986. p. 241-258.

BOURDIEU, P.; PASSERON, J.-C. Reproduction in education, society and culture. London: Sage, 1977.

BRASIL. Ministério da Educação. Instituto Nacional de Estudos e Pesquisas Educacionais (INEP). Censo da Educação Superior 2010. Brasília, DF: Inep, 2011.

BREEN, R. et al. Non persistent inequality in educational attainment: evidence from eight European countries. American Journal of Sociology, Chicago, v. 114, p. 1475-1521, 2009.

CAMERON, S.V.; HECKMAN, J.J. Life cycle schooling and dynamic selection bias: models and evidence for five cohorts of American males. Journal of Political Economy, Chicago, v. 106, p. 262-333, 1998.

CASTRO, M.H.M. The state and the market in the regulation of higher education in Brazil. In: BROCK, C.; SCHWARTZMAN, S. (Ed.). The challenges of education in Brazil. Oxford: Symposium, 2004. p. 179-208.

COLLARES, A.C.M. Educational inequalities and the expansion of postsecondary education in Brazil, from 1982 to 2006. 2010. 304f. Tese (Doutorado em Sociologia) - Departamento de Sociologia, Universidade de Wisconsin, Madison.

CORBUCCI, P. Sobre a redução das matrículas no ensino médio regular. Brasília, DF: IPEA, 2009. (Texto para Discussão, n. 1421).

DOWNEY, D. When bigger is not better: family size, parental resources, and children's educational performance. American Sociological Review, Aliso Viejo, v. 60, p. 746-761, 1995.

DURHAM, E.R. Higher education in Brazil: public and private. In: BROCK, C.; SCHWARTZMAN, S. (Ed.). The challenges of education in Brazil. Oxford: Symposium, 2004. p. 147-168.

ERIKSON, R.; GOLDTHORPE, J.H. The constant flux: a study of class mobility in industrial societies. Oxford: Clarendon, 1992. 
ERIKSON, R.; JONSSON, J.O. Explaining class inequality in education: the Swedish test case. In: ERIKSON, R.; JONSSON, J.O. (Eds.). Can education be equalized?. Boulder: Westview, 1996. p. 1-64.

FERNANDES, D.C. Educational stratification, race and socioeconomic development in Brazil. 1999. 203f. Tese (Doutorado em Sociologia) - Departamento de Sociologia, Universidade de Wisconsin, Madison.

FRANK, D.J.; MEYER, J.W. Worldwide expansion and change in the university. In: KRUCHEN, G.; KOSMUTZKY, A.; TORKA, M. (Ed.). Towards a multiversity?: universities between global trends and national traditions. Bielefeld: Transcript, 2006. p. 19-44.

INSTITUTO BRASILEIRO DE GEOGRAFIA E ESTATÍSTICA (IBGE). Pesquisa nacional por amostra de domicílios - 1982, 2001 a 2009. Microdados. Rio de Janeiro: IBGE, 2012.

INSTITUTO BRASILEIRO DE GEOGRAFIA E ESTATÍSTICA (IBGE). Censo demográfico - 1991, 2000 e 2010. Microdados. Rio de Janeiro: IBGE, 2012.

MAGGIE, Y.; FRY, P. A reserva de vagas para negros nas universidades brasileiras. Estudos Avançados, São Paulo, v. 18, p. 93-117, 2004.

MARE, R.; CHANG, H.-C. Family attainment norms and educational stratification in the United States and Taiwan: the effects of parents' school transitions. In: MORGAN, S.L.; GRUSKY, D.B.; FIELDS, G.S. (Ed.). Mobility and inequality: frontiers of research in Sociology and Economics. Stanford: Stanford University, 2006. p. 195-231.

MARTINS, C.B. A reforma universitária de 1968 e a abertura para o ensino superior privado no Brasil. Educação \& Sociedade, Campinas, v. 30, n. 106, p. 15-35, 2009.

MONT'ALVÃO, A. Estratificação educacional no Brasil do século XXI. Dados: Revista de Ciências Sociais, Rio de Janeiro, v. 54, p. 389-430, 2011.

MONT'ALVÃO, A. Estratificação do acesso ao ensino superior no Brasil. 2013. 257f. Tese (Doutorado em Sociologia) - Programa de Pós-Graduação em Sociologia, Universidade Federal de Minas Gerais, Belo Horizonte.

NEVES, C.E.B. Diversificação do sistema de educação terciária: um desafio para o Brasil. Tempo Social, São Paulo, v. 15, p. 21-44, 2003.

PARSONS, T. Equality and inequality in modern society, or social stratification revised. In: LAUMANN, E.O. (Ed.). Social stratification: research and theory for the 1970s. Indianapolis: Bobbs-Merril, 1970. p. 13-72.

PASTORE, J.; SILVA, N.V. Mobilidade social no Brasil. Rio de Janeiro: Topbooks, 2000.

PRATES, A.A.P. Os sistemas de ensino superior na sociedade contemporânea: diversificação, democratização e gestão organizacional - o caso brasileiro. 2005. 251f. Tese (Doutorado 
em Sociologia) - Programa de Pós-Graduação em Sociologia, Universidade Federal de Minas Gerais, Belo Horizonte.

REIS, F.W. Democracia, universidade e relações raciais. In: PEIXOTO, M.C.L. (Org.). Universidade e democracia: experiências e alternativas para a ampliação do acesso à universidade pública brasileira. Belo Horizonte: UFMG, 2004. p. 21-32.

RIBEIRO, C.A.C. Estrutura de classes, condições de vida e oportunidades de mobilidade social no Brasil. In: SILVA, N.V.; HASENBALG, C. (Ed.). Origens e destinos: desigualdades sociais ao longo da vida. Rio de Janeiro: Topbooks, 2003. p. 381-425.

RIBEIRO, C.A.C. Desigualdade de oportunidades no Brasil. Belo Horizonte: Argumentum, 2009. p. 21-74.

RIBEIRO, C.A.C. Desigualdade de oportunidades e resultados educacionais no Brasil. Dados: Revista de Ciências Sociais, Rio de Janeiro, v. 54, p. 41-87, 2011.

SCHOFER, E.; MEYER, J.W. The worldwide expansion of higher education in the twentieth century. American Sociological Review, Aliso Viejo, n. 70, 898-920, 2005.

SCHWARTZMAN, S. Equity, quality and relevance in higher education in Brazil. Anais da Academia Brasileira de Ciências, Rio de Janeiro, v. 76, p. 173-188, 2004.

SCHWARTZMAN, S. A expansão do ensino superior, a sociedade do conhecimento e a educação tecnológica. São Paulo: Senai; Departamento Nacional, 2005.

SHAVIT, Y.; BLOSSFELD, H.-P. Persistent inequality: a comparative study of educational attainment in thirteen countries. Boulder: Westview, 1993.

SHAVIT, Y.; ARUM, R.; GAMORAN, A. Stratification in higher education: a comparative study. Palo Alto: Stanford University, 2007.

SILVA, N.V. Expansão escolar e estratificação educacional no Brasil. In: SILVA, N.V.; HASENBALG, C. (Ed.). Origens e destinos: desigualdades sociais ao longo da vida. Rio de Janeiro: Topbooks, 2003. p.105-138.

SILVA, N.V.; HASENBALG, C. Tendências da desigualdade educacional no Brasil. Dados: Revista de Ciências Sociais, Rio de Janeiro, v. 43, p. 423-445, 2000.

SILVA, N.V.; HASENBALG, C. Recursos familiares e transições educacionais. Cadernos de Saúde Pública, Rio de Janeiro, v. 18, p. 67-76, 2002.

SOARES, J.F.; ALVES, M.T.G. Desigualdades raciais no sistema brasileiro de educação básica. Educação \& Pesquisa, São Paulo, v. 29, p. 147-165, 2003.

TAM, T. Accounting for dynamic selection bias in educational transitions: the Cameron-Heckman latent class estimator and its generalizations. Research in Social Stratification and Mobility, v. 29, p. 287-310, 2011. 
TORCHE, F. Privatization reform and inequality of educational opportunity: the case of Chile. Sociology of Education, Albany, v. 78, p. 316-343, 2005.

TREIMAN, D.J. Industrialization and social stratification. In: LAUMANN, E.O. (Ed.). Social stratification: research and theory for the 1970s. Indianapolis: BobbsMerrill, 1970. p. 207-234.

Recebido em 22 de abril de 2013.

Aprovado em 20 de agosto de 2013. 